\title{
Results of the implementation of a virtual microscope in a course of histology
}

\author{
Alegre-Martínez, Antoni ${ }^{a}$; Martínez-Martínez, M${ }^{\mathrm{a}}$ Isabel $^{\mathrm{b}}$; Alfonso Sánchez, José \\ Luis $^{c}$; Morales Suarez-Varela, Maria M. ${ }^{\text {; LLopis González, Agustín }}{ }^{\text {d }}$ \\ ${ }^{a}$ Departamento de Ciencias Biomédicas, Universidad Cardenal Herrera CEU. Avenida \\ Seminario, s/n, 46113 Montcada, Valencia (SPAIN), 'Facultad de Enfermería y Podología, \\ Universidad de Valencia. Carrer de Jaume Roig, s/n, 46001 València, (SPAIN), ' Facultad \\ de Medicina, Universidad de Valencia. Av. de Blasco Ibáñez, 15, 46010 València, \\ (SPAIN), ' Facultad de Farmacia, Universidad de Valencia. Carrer del Dr. Moliner, 50, \\ 46100 Burjassot, Valencia (SPAIN).
}

\begin{abstract}
The course of Anatomy and Histology is studied in the first year of Dentistry at the University Cardenal Herrera CEU (Alfara del Patriarca, Spain). Its practices consist on choose freely six different tissue samples and draw their most representative features. These practices were made by optical microscopy until 2014, and in 2015 was introduced the virtual microscope exclusively. The aim of the study is to test whether this new teaching method has improved the quality of exercise and the understanding shown by students. First, the best exercises of both years were chosen, and from them some drawings from the same tissue were compared. Some tissues which samples for optical microscope were hard to obtain, were drawn for the very first time thanks to the virtual microscope. Also, with the virtual microscopy the drawings contained more details and definition. The understanding of the structures improved, shown by a more functional, detailed and defined vision of the tissues. The labels of the virtual microscope helped to the self-study and avoided the loss of unnoticed structures. In conclusion, replacement of optical microscope by the virtual microscope is a teaching improvement and facilitates student learning.
\end{abstract}

Keywords: Virtual; Optical; Microscope; Teaching; Histology; Improvement 


\section{Introduction}

Virtual slides are digital reproductions of optical microscope slides that can be viewed by a software that can emulate a traditional microscope (Dee, Lehman, Consoer, Leaven, \& Cohen, 2003). These slides can be explored in a web browser that closely simulates examination of real glass slides with an optical microscope (Kumar et al., 2004). The technology for production of virtual slides was developed in 1985; but was in the late 1990s when desktop computers had enough processing speed to expand virtual microscopy and use this technology for education. As a result, by 2000, the use of the optical microscopy in medical education had decreased for allow the entry of virtual microscope in the faculties (Dee, 2009). In 2001 Bacus patented a system for fast scan of microscope samples and turn them into virtual slides (Bacus \& Bacus, 2001). In 2009, about 50\% of pathology courses in United States already have or expect to implement virtual microscopy (Dee, 2009). The virtual microscope has advantages and disadvantages respect to the optical microscope. However, the student preferences for one or other microscope change widely (Scoville \& Buskirk, 2007).

One of the main advantages is the saving cost: the optical microscopes and glass slides are expensive and require maintenance and reparations (Dee, 2009). The virtual microscope only needs a computer or tablet with a web browser (Rehatschek \& Hye, 2011). Also, the single-use microscope laboratory can be converted into a multiuse computer laboratory (Dee, 2009). Other advantages cited are that the virtual microscope is easy to learn, and students and professors adapt very quickly to the use of the virtual microscope (Kumar et al., 2004). Respect to the higher resolution, the slides provides excellent image quality (Kumar et al., 2004) in part thanks to the improvement of the streaming (Afework et al., 1998) Virtual slides are always focused and with an adequate level of light adjustment (Harris et al., 2001). Also the exploration of the sample is much better in the virtual microscope: virtual slides enhance the ability of the students to grasp and explore morphological features better than optical microscope (Dee et al., 2003). They are able to magnify, scroll and take pictures of images of their samples for use them for their notes or presentations (Goldberg \& Dintzis, 2007).

The use of labels in the virtual microscope allows students to find tumours o growths (Afework et al., 1998). The students can write their own notes in the slide, which helps them to understand deeper the sample (Goldberg \& Dintzis, 2007). Other advantage is the standardization of the content, because all students are exposed to the same samples. The virtual microscope avoids the problem of maintaining a satisfactory assortment of glass slides, which are easy to break (Goldberg \& Dintzis, 2007). In addition, the virtual microscope allows the group study being able to point with a finger to features on virtual slides, allowing interaction tutor-student or between students (Randell et al., 2012) and allowing to focus on the links between histology, physiology, and pathology (Goldberg \& 
Dintzis, 2007). It has been studied that the use of virtual microscope reduce the learning time: students learn more quickly (Harris et al., 2001) and the time required for a concrete lesson can be reduced to a half (Heidger et al., 2002). The average marks of the practical exams also increase (Goldberg \& Dintzis, 2007). Other of the advantages is that the virtual microscope is always available: students can review the samples at any time, not only when the microscopy laboratory is open(Goldberg \& Dintzis, 2007) This is useful especially preceding practical examinations (Harris et al., 2001). Also, the students can use additional features of the new media, such to share the findings of the slide to the community or in social networks (Rehatschek \& Hye, 2011). Also professors can take advantage of the accessibility, efficiency, and pedagogic versatility of the computer and the Internet (Dee, 2009). In the future is expected the introduction of the virtual microscope using a Powerwall, a high-resolution array of 28 computer screens (Treanor et al., 2009).

However, the virtual microscope also has some disadvantages. To digitalize a collection of slides for the first time is expensive and requires investment (Goldberg \& Dintzis, 2007). It is a problem to store and process the extremely large quantity of data required for a collection of virtual slides (Çatalyürek et al., 2003). A single slide consists of 5 to 30 focal planes, with about 7GB each one, which means a total of 35-210GB per slide (Ferreira et al., 1997). The speed can also be an obstacle: depending on the speed of the Internet connection, there can be a large latency in processing these data when changing zoom or browsing the sample (Ferreira et al., 1997), especially in periods of high peak demand (Kumar et al., 2004). Other problems are related to the public use: Nowadays, only few virtual microscopes for public use can be found on the web, perhaps due to a lack of solutions at reasonable cost (Glatz-Krieger, Glatz, \& Mihatsch, 2003). In the future it is need to work in the development of a public domain set of virtual slides (Harris et al., 2001). For ending, there is the problem of the oblivion of the optical microscope. Students must have the chance to learn techniques of traditional microscopy, maintaining some of them in the laboratories (Goldberg \& Dintzis, 2007).

\section{Objectives}

The course of Anatomy and Histology is studied in the first year of Dentistry at the University Cardenal Herrera CEU (Alfara del Patriarca, Spain). The practices were made by optical microscopy until 2014. In them, following an explanation by the teacher, students must choose freely six samples and draw their most representative features. In 2015, for the first time, the practice was made using only the virtual microscope histologyguide.org created by professors Robert L. Sorenson PhD y T. Clark Brelje PhD. The aim of the study is to test whether this new teaching method has improved the quality of exercise and the understanding shown by students. 


\section{Material and methods}

The first step is to choose the best exercises performed by students of the Anatomy and Histology course in 2014 and 2015. The second step is to choose from these exercises, some drawings for compare them and observe the main changes between both microscopes and obtain conclusions.

\section{Results}

First, it is very notorious that in 2015 the students chose tissues that were not drawn up until now, because normally they are very difficult to obtain or the samples are fragile. Thus, as shown in Figure 1, students chose brand new samples such compact bone or eye thanks to their availability in virtual microscope. It is also very evident that the student drew both tissues with a high level of detail.

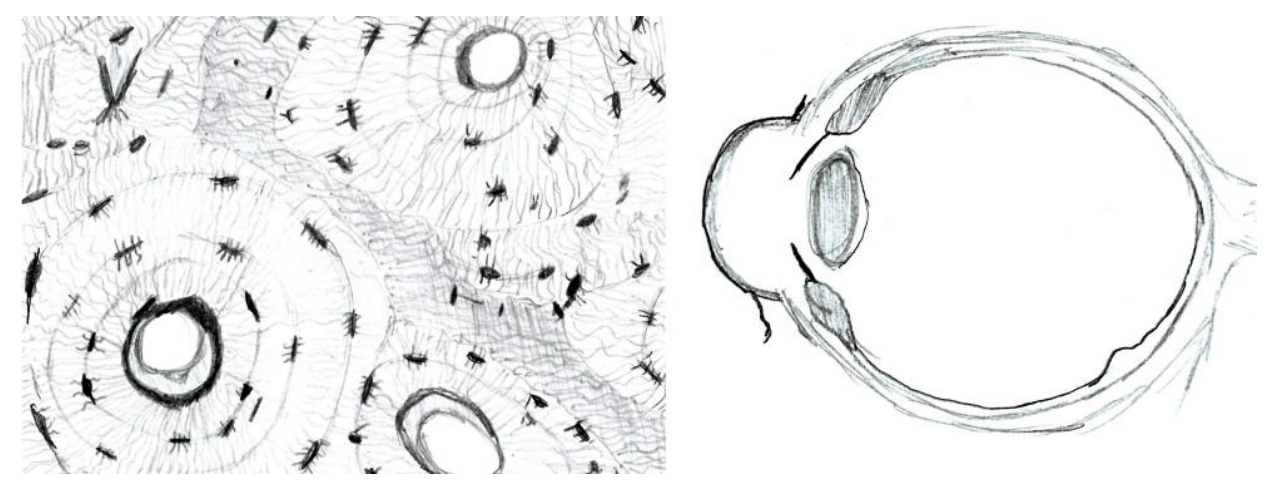

Figure 1. Compact bone on the left, and eye on the right, both with virtual microscope 2015.

In Figure 2 it is compared the renal cortex drawn using an optical microscope and a virtual one. It shows how the student using an optical microscope drew the characteristic rounded contour, a classic detail that is lost in the virtual microscope. Because the optical microscope used in the practice cannot achieve more than 50x magnification, the Bowman's capsule is not appreciated in detail, so the student interpreted it as a glomerulus with random nuclei inside. The level of detail that shows the virtual microscope is much higher, appreciating all the contours of the cells and their nuclei. In this way, students could see more clearly the structure of the renal cortex. 

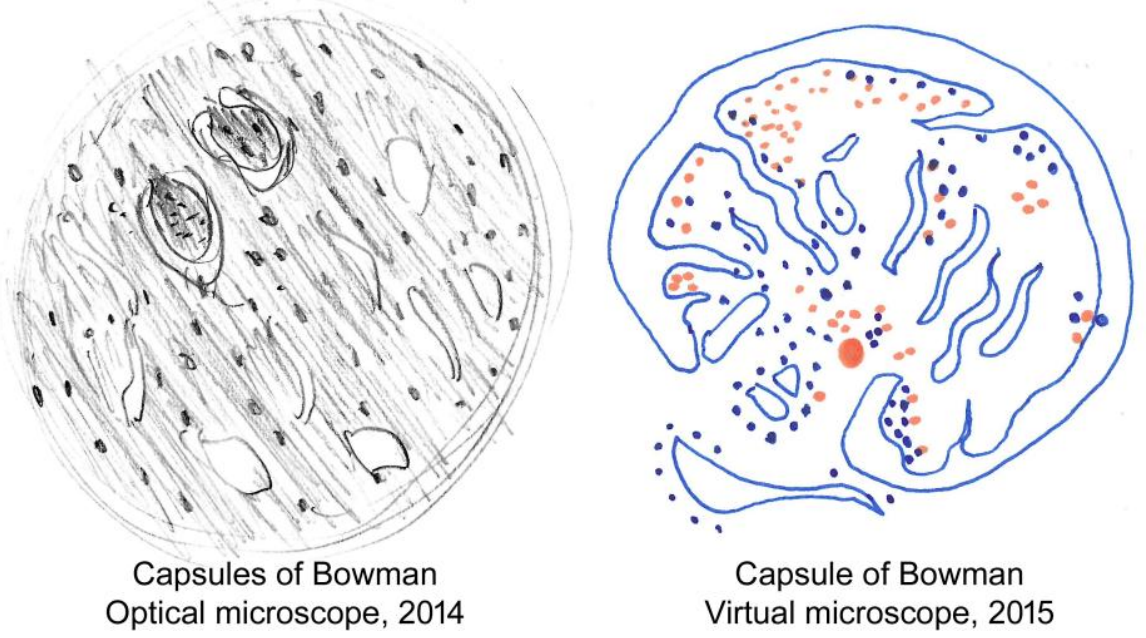

Figure 2. Comparison of two drawings of the renal cortex.

In Figure 3 a sample of skeletal muscle is shown. Watching through the optical microscope, the muscle fibers intersect and the student has the impression that they form a tangle, putting the focus in the striations and rounded nuclei. Using the virtual microscope, the student drew every fibre separately and also realized that the nuclei are elongated and not round.

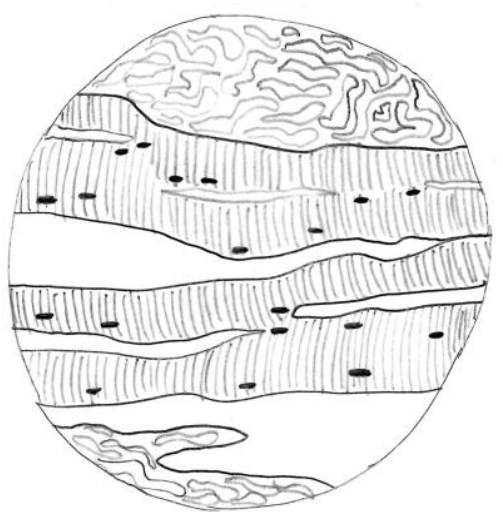

Skeletal muscle Optical microscope, 2014

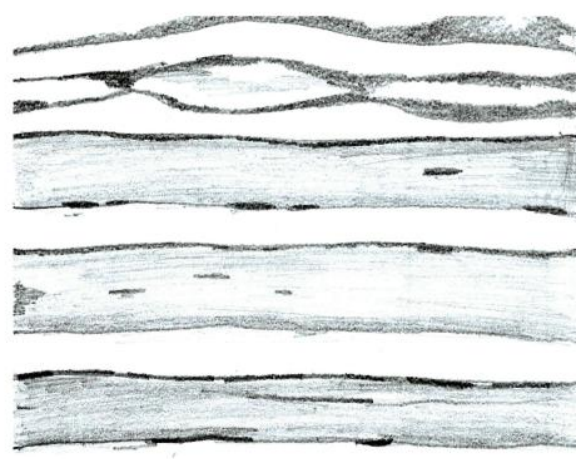

Skeletal muscle Virtual microscope, 2015

Figure 3. Comparison of two drawings of skeletal muscle. 
In Figure 4, a sample of intestinal microvilli are observed. This sample is hard to prepare because it is very difficult to make a longitudinal section of the villi. In fact, in the picture of the left the villi are cut axially, making it difficult for the student to understand the villi as fingerings from the mucosa. In the professional virtual slide, the cut is perfectly longitudinal, and the student can draw the structure of the villi with great precision and understanding its whole structure.

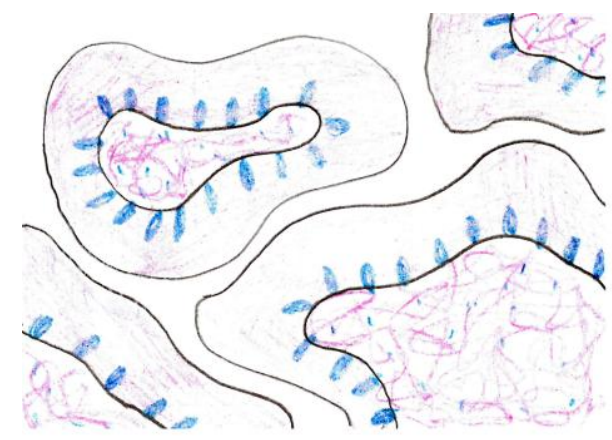

Intestinal villi Optical microscope, 2014



Intestinal villi Virtual microscope, 2015

Figure 4. Comparison of two drawings of the small intestine.

The Figure 5 is a sample of tongue. Due to the absence of labels, the student who used the optical microscope focused on the oral mucosa, drawing the stratified squamous nonkeratinized epithelium that was the most striking thing of the sample due to its intense red colour. However, the student who used the virtual microscope, aided by labels, noticed the huge number of structures that are in the tongue, drawing many papillae and numerous serous glands. Despite this self-study, the teacher should continuously check the development of the exercise and resolve doubts.
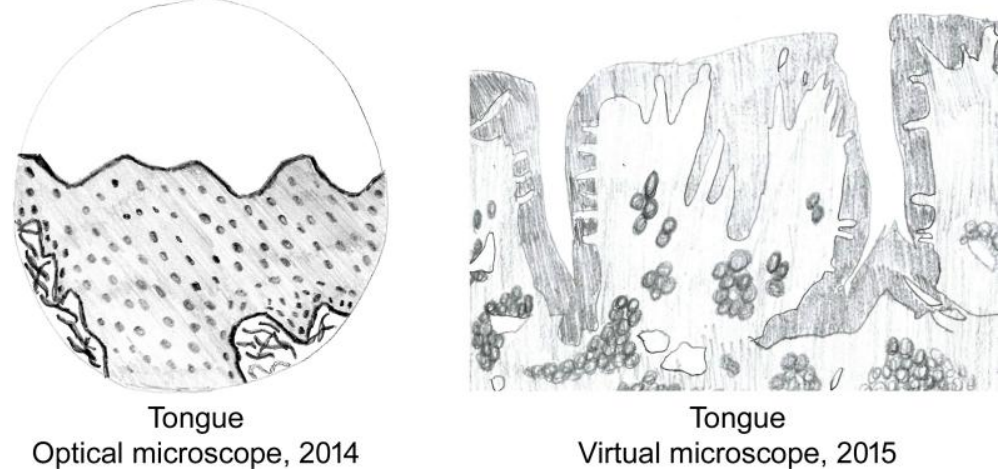

Figure 5. Comparison of two drawings of the tongue. 


\section{Conclusions}

The replacement of the optical microscope by the virtual microscope in the courses of histology not only implies an improvement in costs, also results in a better understanding of the samples by the students, helps them to have a more functional, detailed and defined vision of the tissues. Thanks to the labels they can learn by themselves the main landmarks of the sample and not to miss unnoticed structures. It also allows the learning of organs and tissues which samples are difficult to prepare for the optical microscopy.

\section{References}

Afework, A., Beynon, M. D., Bustamante, F., Cho, S., Demarzo, A., Ferreira, R., Tsang, H. (1998). Digital dynamic telepathology--the virtual microscope. Proceedings / AMIA .Annual Symposium.AMIA Symposium, , 912-916.

Bacus, J. V., \& Bacus, J. W. (2001). Method and Apparatus for Creating a Virtual Microscope Slide,

Çatalyürek, Ü, Beynon, M. D., Chang, C., Kurc, T., Sussman, A., \& Saltz, J. (2003). The virtual microscope. Information Technology in Biomedicine, IEEE Transactions On, 7(4), 230-248.

Dee, F. R. (2009). Virtual microscopy in pathology education. Human Pathology, 40(8), 1112-1121.

Dee, F. R., Lehman, J. M., Consoer, D., Leaven, T., \& Cohen, M. B. (2003). Implementation of virtual microscope slides in the annual pathobiology of cancer workshop laboratory. Human Pathology, 34(5), 430-436.

Ferreira, R., Moon, B., Humphries, J., Sussman, A., Saltz, J., Miller, R., \& Demarzo, A. (1997). The virtual microscope. Proceedings : A Conference of the American Medical Informatics Association / ...AMIA Annual Fall Symposium.AMIA Fall Symposium, , 449-453.

Glatz-Krieger, K., Glatz, D., \& Mihatsch, M. J. (2003). Virtual slides: High-quality demand, physical limitations, and affordability. Human Pathology, 34(10), 968-974.

Goldberg, H. R., \& Dintzis, R. (2007). The positive impact of team-based virtual microscopy on student learning in physiology and histology. Advances in Physiology Education, 31(3), 261-265. doi:31/3/261 [pii]

Harris, T., Leaven, T., Heidger, P., Kreiter, C., Duncan, J., \& Dick, F. (2001). Comparison of a virtual microscope laboratory to a regular microscope laboratory for teaching histology. The Anatomical Record, 265(1), 10-14. 
Heidger, P. M., Dee, F., Consoer, D., Leaven, T., Duncan, J., \& Kreiter, C. (2002). Integrated approach to teaching and testing in histology with real and virtual imaging. The Anatomical Record, 269(2), 107-112.

Kumar, R. K., Velan, G. M., Korell, S. O., Kandara, M., Dee, F. R., \& Wakefield, D. (2004). Virtual microscopy for learning and assessment in pathology. The Journal of Pathology, 204(5), 613-618.

Randell, R., Hutchins, G., Sandars, J., Ambepitiya, T., Treanor, D., Thomas, R., \& Ruddle, R. (2012). Using a high-resolution wall-sized virtual microscope to teach undergraduate medical students. CHI'12 Extended Abstracts on Human Factors in Computing Systems, 2435-2440.

Rehatschek, H., \& Hye, F. (2011). The introduction of a new virtual microscope into the eLearning platform of the medical university of graz. Interactive Collaborative Learning (ICL), 2011 14th International Conference On, 10-15.

Scoville, S. A., \& Buskirk, T. D. (2007). Traditional and virtual microscopy compared experimentally in a classroom setting. Clinical Anatomy, 20(5), 565-570.

Treanor, D., Jordan- Owers, N., Hodrien, J., Wood, J., Quirke, P., \& Ruddle, R. A. (2009). Virtual reality powerwall versus conventional microscope for viewing pathology slides: An experimental comparison. Histopathology, 55(3), 294-300. 